\title{
Bromocriptine Use in Peripartum Cardiomyopathy: Review of Cases
}

\author{
Rebecca Simon, MD ${ }^{1}$ Sophia Yang, $\mathrm{MS}^{1} \quad$ Afshan B. Hameed, MD \\ ${ }^{1}$ Irvine Department of Obstetrics and Gynecology, University of \\ California, Orange, California \\ Am J Perinatol Rep 2018;8:e335-e342.

\begin{abstract}
Address for correspondence Afshan B. Hameed, MD, Irvine Department of Obstetrics and Gynecology, University of California, 333 City Blvd W, Suite 1400, Orange, CA 92868 (e-mail: ahameed@uci.edu).
\end{abstract}

\begin{abstract}
Keywords

- peripartum cardiomyopthaty

- bromocriptine

- left ventricular function

- recovery

- pregnancy

- right ventricular function

Objective This study is to review published cases of peripartum cardiomyopathy (PPCM) treated with bromocriptine and outline pros and cons of the treatment strategy.

Data Sources Data were collected from PubMed/MedLine, ClinicalTrials.gov; the years 2007 to 2018 were searched for English-language articles. Search terms: "bromocriptine and peripartum cardiomyopathy", "bromocriptine and cardiomyopathy."

Methods of Study Selection This search strategy yielded 171 articles. After excluding duplicates, 86 studies were reviewed. Sixty-one articles involving the treatment of PPCMP were included, and of these, 17 were case reports of patients with PPCMP treated with bromocriptine; these studies were included in this review.

Tabulation, Integration, and Results Seventeen of these articles were case reports of patients with peripartum cardiomyopathy treated with bromocriptine that were included. Conclusion Bromocriptine seems to be a promising treatment, there is currently insufficient evidence for universal utilization of bromocriptine for all patients with PPCMP. Addition of bromocriptine to the standard heart failure therapy should be individualized.
\end{abstract}

Peripartum cardiomyopathy (PPCM) is a rare but potentially devastating form of cardiomyopathy occurring late in pregnancy or early postpartum period in previously healthy women. ${ }^{1}$ Pregnancy associated heart failure was first described in the 1800s; however it was not until 1971 that Demakis and Rahimtoola who recognized the disease as a distinct entity and coined the term peripartum cardiomyopathy. ${ }^{1-3}$ According to the 2010 European Society of Cardiology (ESC), diagnosis of PPCM is made by echocardiography demonstrating ejection fracture of $<45 \%$ with or without the left ventricular dilation with no evidence of other potential etiologies of heart failure. ${ }^{4}$ Our goal is to provide brief overview of PPCM, review published cases of PPCM treated with bromocriptine and outline pros and cons of the treatment strategy.

\section{Incidence and Risk Factors}

Incidence of PPCM in the United States varies widely from 1 in 1,000 to 1 in 4,000 live births. ${ }^{5}$ The risk of PPCM is largely

received

October 9, 2017

accepted after revision

September 12, 2018 influenced by ethnicity with African-American women at highest risk followed by Asians, whites, and Hispanic women. ${ }^{6}$ Geographically, the highest incidence is encountered in Haiti (1 in 300 pregnancies) and South Africa ( 1 in $1,000$ pregnancies $).^{7-9}$ Other risk factors include multiparity, multifetal gestation, preeclampsia, gestational hypertension, and advanced maternal age. ${ }^{8}$ In fact, greater than $50 \%$ of cases occur in women older than 30 years old. ${ }^{1}$

\section{Treatment Options for PPCM}

Treatment of PPCM is similar to other types of heart failure with reduced ejection fraction. Mainstay of therapy is salt and fluid restriction, diuretics, vasodilators, and beta blockers. Anticoagulation may be indicated in selected cases. However, the use of angiotensin converting enzyme inhibitors (ACE) and angiotensin receptor blockers, (ARB) which have been shown to reduce morbidity and mortality are deferred until after delivery. ${ }^{7,10,11}$

Copyright $\odot 2018$ by Thieme Medical Publishers, Inc., 333 Seventh Avenue, New York, NY 10001, USA. Tel: +1(212) 584-4662.
License terms

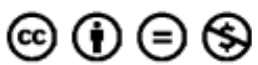

DOI https://doi.org/

10.1055/s-0038-1675832. ISSN 2157-6998. 


\section{Natural History of PPCM and Outcomes}

Overall, outcomes of PPCM tend to be favorable compared to other types of cardiomyopathies as it is less likely to progress to end stage than heart failure caused by other etiologies. ${ }^{12}$ With current treatment modalities, PPCM patients have a $50 \%$ rate of recovery and $98 \%$ chance of survival. ${ }^{13}$ Previously, it was thought that if PPCM were to resolve, it would do so within 6 months of diagnosis and it persisted past this time point; it was considered a poor prognostic factor. ${ }^{2}$ However, more recently, Fett et al followed 116 Haitian patients with PPCM and only $27.6 \%$ achieved full recovery; of those who recovered, $53 \%$ did not achieve full recovery for at least 18 months. ${ }^{14}$ While this may be specific to the Haitian population, it appears that this disease may take a more significant amount of time to resolve than previously thought. Further, the risk of recurrence of PPCM is high and those who have had a pregnancy complicated by it are counseled to avoid future pregnancy. ${ }^{15}$

\section{Theories for Causation}

The exact etiology of PPCM remains unknown; however, significant advances have been made to elucidate causation of PPCM. The largest umbrella of hypotheses include the "oxidative stress-prolactin axis" and "antiangiogenic-signaling excess" hypotheses. ${ }^{7}$ The "oxidative stress-prolactin axis" hypothesis stems from the elevated markers of inflammation and apoptosis found in PPCM. ${ }^{16,17}$ A transgenic mouse model of PPCM was developed to investigate potential mechanisms of the disease. Using this mouse model, Sliwa et al showed that oxidative stress allows expression and activation of a lysosomal enzyme, cathepsin D which cleaves serum prolactin into an antiangiogenic and pro apoptotic $16-\mathrm{kDa}$ prolactin sub fragment that incite and propagates myocardial damage. ${ }^{18}$ The study also showed that blocking the release of prolactin inhibited degeneration of the cardiac capillary network, thereby decreasing myocyte damage. ${ }^{18}$ Another study by Forster at al showed that increased levels of both prolactin and interferongamma were associated with increased inflammatory status and adverse outcomes in PPCM. ${ }^{19}$ Recent data show that the "oxidative stress-prolactin axis" and the "antiangiogenic-signaling excess" probably converges in a final pathway of imbalanced cardiac remodeling in the peripartum phase, thereby causing myocardial injury secondary to metabolic "shortages." 8 Other proposed causative factors which will not be described here, include: selenium deficiency, viral myocarditis, and immune mediated cardiac damage.

\section{Bromocriptine as a Therapy for PPCM}

Bromocriptine is an ergot derivative with dopamine agonistic activity that inhibits the release of prolactin from the anterior pituitary. It is FDA approved for the treatment of hyperprolactinemia-associated endocrine dysfunction, acromegaly, Parkinson's disease, and to improve glycemic control in type 2 diabetes mellitus. In the past, it has also been used to inhibit lactation when medically indicated. Given the evidence to support the oxidative stress-prolactin hypothesis of PPCM, bromocriptine has been introduced as a potential beneficial addition to standard the treatment for PPCM.

Since the publications of the oxidative stress-prolactin axis model, there has been significant interest in the use of bromocriptine for prolactin inhibition in PPCMP cases demonstrating a positive impact on left ventricular ejection fraction and NYHA (New York Heart Association) class. However, bromocriptine is not without risks. Serious adverse events have been reported in postpartum women using bromocriptine for lactation suppression including myocardial infarction, seizures, and stroke. ${ }^{20}$ Among patients with adverse events after bromocriptine, many events may have been avoided if treatment was discontinued with the initial manifestations of adverse reaction. ${ }^{21}$ While a causal relationship remains unclear, routine use of bromocriptine for prevention of physiologic lactation is not recommended. Cessation of lactation may also pose significant disadvantage to the neonate; however, Sliwa et al showed normal growth and survival of neonates with mothers treated with bromocriptine. ${ }^{22}$ Bromocriptine is contraindicated in women with pregnancy-induced hypertension, as it can worsen blood pressures during pregnancy or postpartum periods. Therefore, the risk to benefit ratio of bromocriptine makes it a poor choice for lactation suppression but may be worth taking the risk of adverse events in PPCMP as it may significantly improve cardiac outcomes (- Table $\mathbf{1}$ ).

\section{Sources}

Authors manually searched PubMed/MedLine and ClinicalTrials.gov for English-language articles written from 2007 to 2018 using the search terms "bromocriptine and peripartum cardiomyopathy," "bromocriptine and cardiomyopathy."

\section{Study Selection}

The Search strategy yielded 171 articles. After excluding duplicates, 86 studies were reviewed. Sixty-one articles involving the treatment of PPCMP were included, and of these, 17

Table 1 Advantages and disadvantages of bromocriptine use in peripartum cardiomyopathy

\begin{tabular}{|l|l|}
\hline Advantages & Disadvantages \\
\hline FDA approved & Lactation suppression \\
\hline $\begin{array}{l}\text { Risk of serious adverse effects may be } \\
\text { avoided with close monitoring }\end{array}$ & $\begin{array}{l}\text { Worsening hypertension and may increase risk of neurologic } \\
\text { events in those with pregnancy induced hypertension }\end{array}$ \\
\hline May improve NYHA functional class at follow up & Reported risk of myocardial infarction \\
\hline May improve systolic and diastolic function & Arterial thromboembolism \\
\hline
\end{tabular}

Abbreviation: FDA, Food and Drug Administration; NYHA, New York Heart Association. 


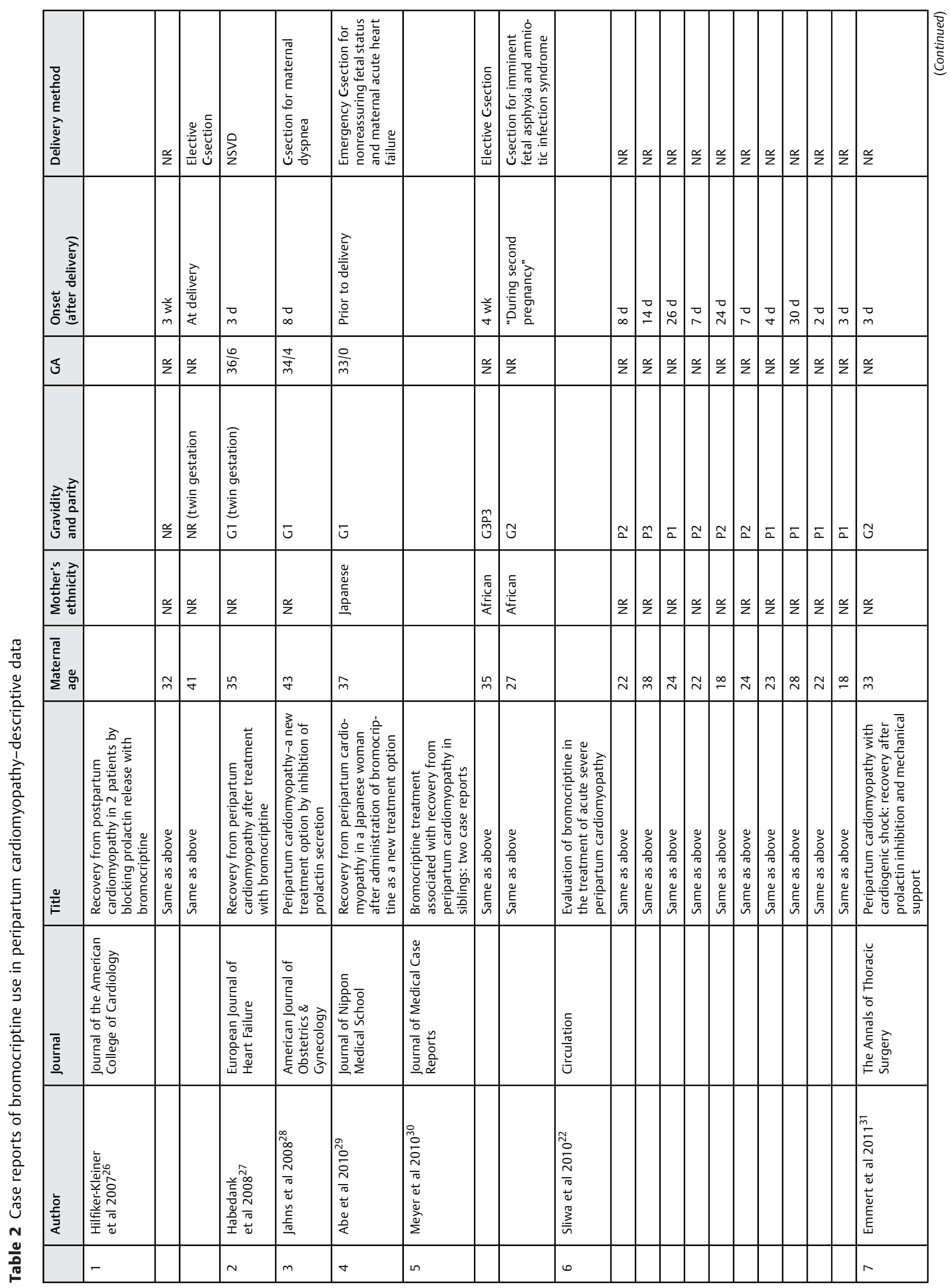




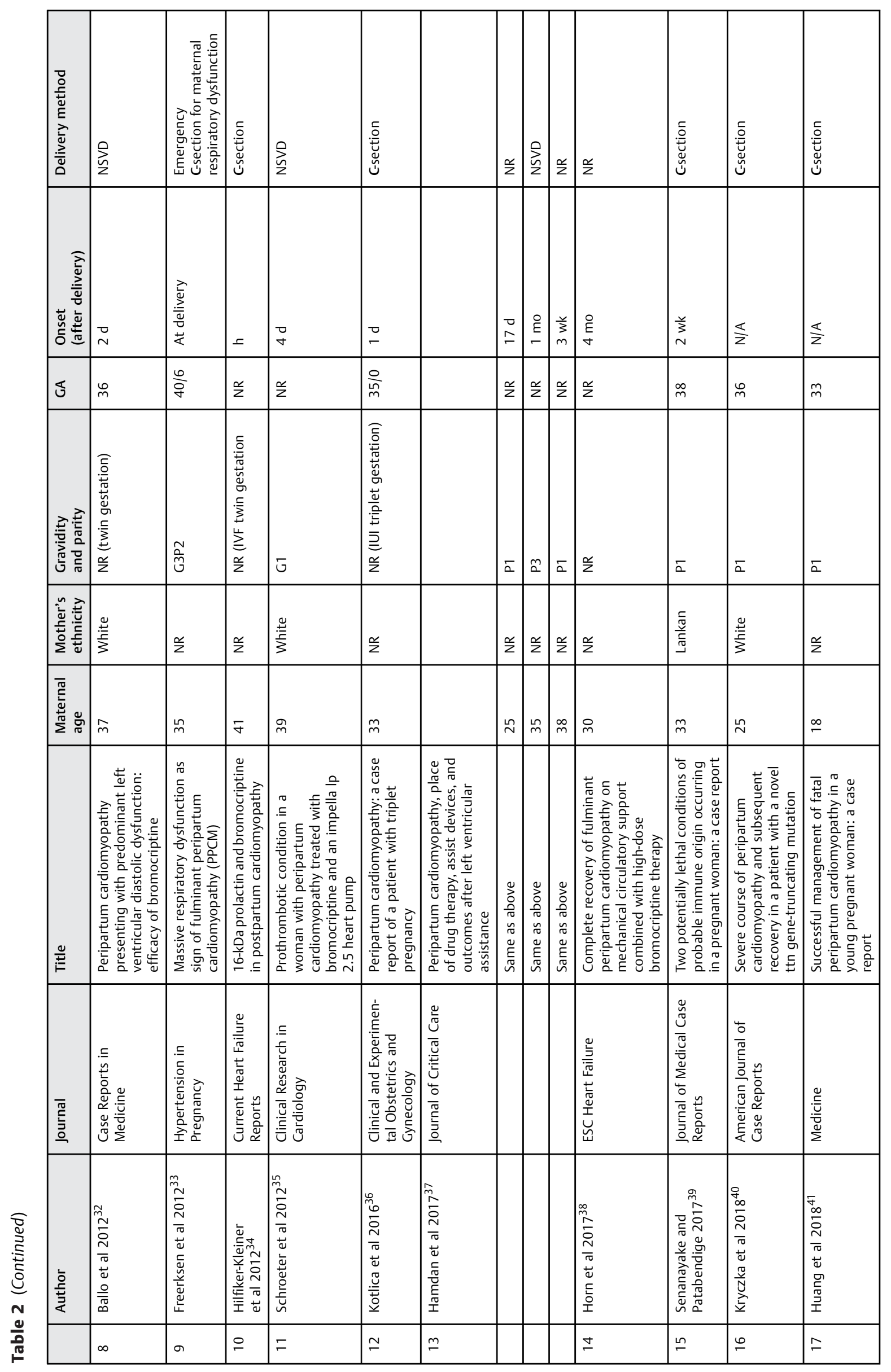

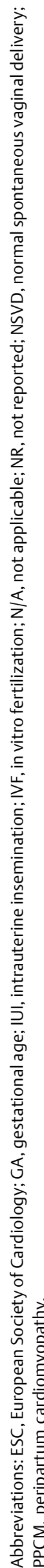


Bromocriptine in Peripartum Cardiomyopathy Simon et al. e339

\begin{tabular}{|c|c|c|c|c|c|c|c|c|c|c|c|c|c|c|c|c|c|c|c|}
\hline 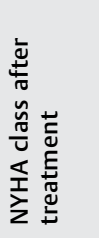 & & 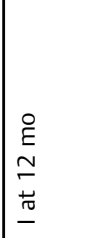 & 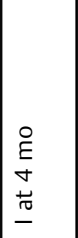 & 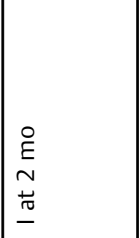 & $\frac{\alpha}{z}$ & $\begin{array}{l}\stackrel{0}{E} \\
m \\
0 \\
\tilde{t} \\
-\end{array}$ & & \begin{tabular}{|l}
$\stackrel{0}{E}$ \\
6 \\
0 \\
$=$ \\
$=$
\end{tabular} & $\frac{\alpha}{z}$ & & $\begin{array}{l}\stackrel{0}{E} \\
6 \\
\stackrel{+}{\Delta} \\
-\end{array}$ & 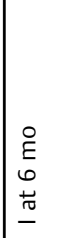 & 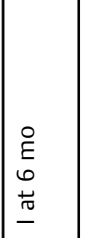 & 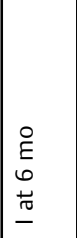 & 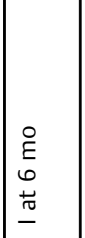 & 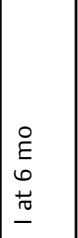 & \begin{tabular}{|l}
0 \\
$E$ \\
0 \\
0 \\
\\
-
\end{tabular} & \begin{tabular}{|l}
0 \\
$E$ \\
0 \\
0 \\
\\
-
\end{tabular} & 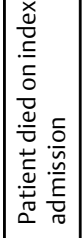 \\
\hline 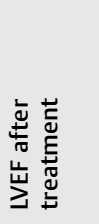 & & 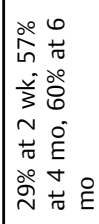 & 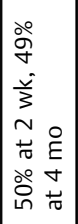 & 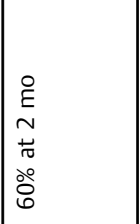 & 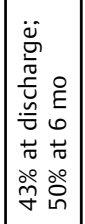 & 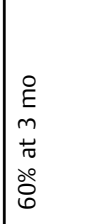 & & 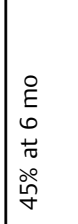 & 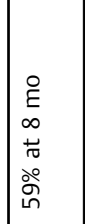 & & ळ & $\stackrel{\circ 0}{\stackrel{\circ}{m}}$ & ঐे & ঐे & 总 & 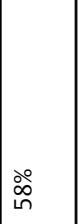 & 今̊ & 迨 & $\frac{\varangle}{z}$ \\
\hline 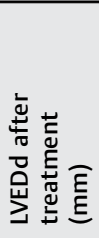 & & 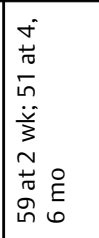 & 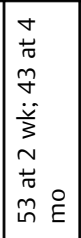 & 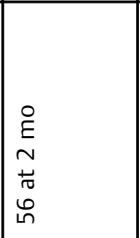 & $\frac{\alpha}{z}$ & 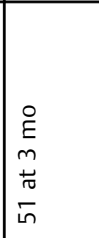 & & $\frac{\alpha}{z}$ & $\frac{o}{z}$ & & $\forall$ & ถి & : & in & $\stackrel{\infty}{\stackrel{9}{+}}$ & in & F & $\dot{m}$ & $\frac{\pi}{z}$ \\
\hline 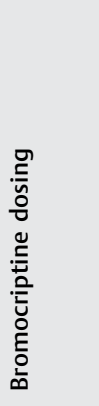 & & 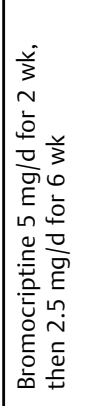 & 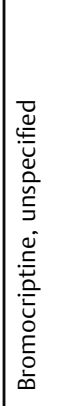 & 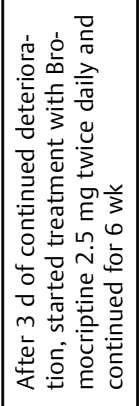 & 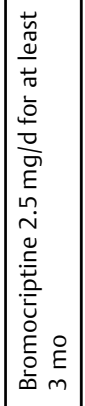 & 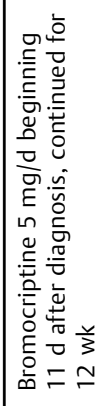 & & 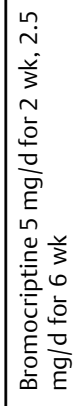 & 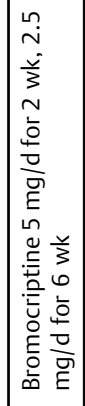 & & 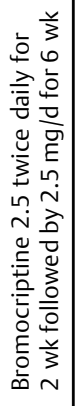 & 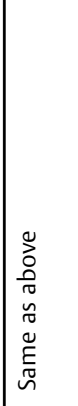 & 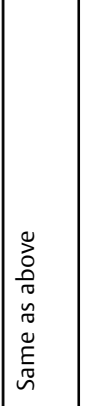 & 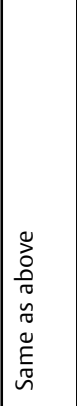 & 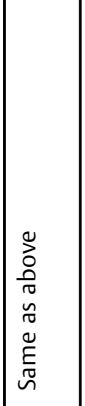 & 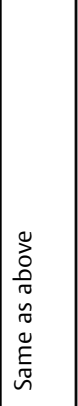 & 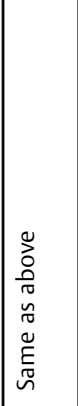 & 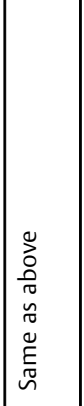 & 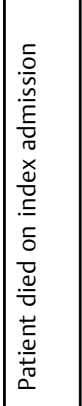 \\
\hline 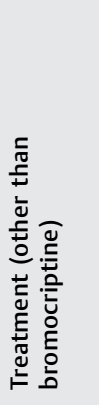 & & 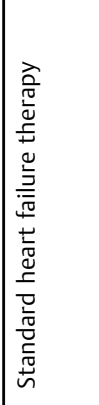 & 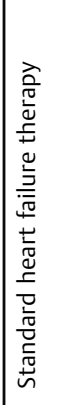 & 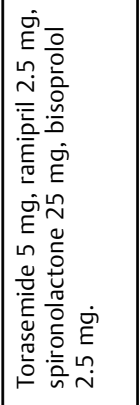 & 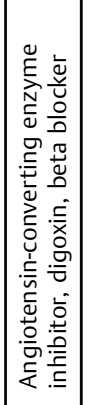 & 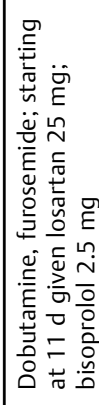 & & 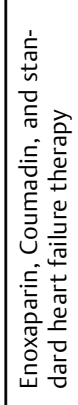 & 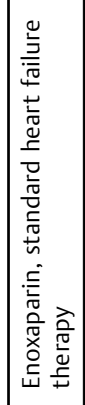 & & 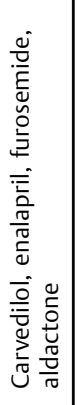 & 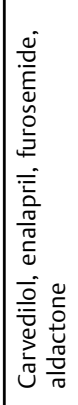 & 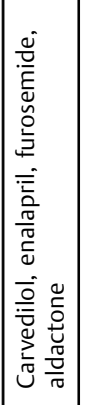 & 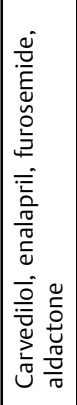 & 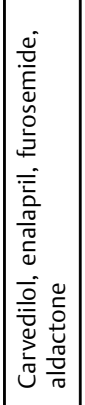 & 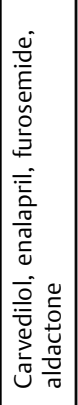 & 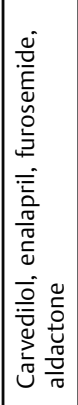 & 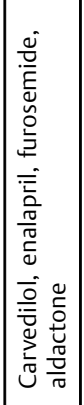 & 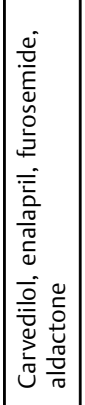 \\
\hline 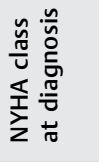 & & $\equiv$ & $\geq$ & $\frac{\tilde{z}}{z}$ & $\frac{\alpha}{z}$ & $=$ & & $\geq$ & $\frac{o}{z}$ & & $\geq$ & $=$ & $=$ & $=$ & $=$ & $\equiv$ & $\geq$ & $=$ & $\equiv$ \\
\hline 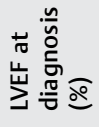 & & 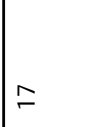 & $\stackrel{\text { m }}{ }$ & $\stackrel{\stackrel{n}{\sim}}{ }$ & $\stackrel{\text { m }}{n^{\prime}}$ & $\mid \begin{array}{l}\stackrel{P}{\bar{N}} \\
\dot{\bar{j}}\end{array}$ & & $a$ & $\tilde{m}$ & & $\stackrel{\vec{m}}{\mathrm{~m}}$ & $\stackrel{\mathscr{N}}{ }$ & $\stackrel{\circ}{m}$ & 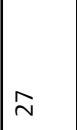 & in & m & $\stackrel{m}{m}$ & $\tilde{m}$ & $\stackrel{\infty}{\sim}$ \\
\hline 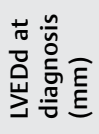 & & 8 & 只 & 8 & 兰 & $\stackrel{\infty}{\circ}$ & & $\tilde{\theta}$ & 8 & & $m$ & ڤి & $\stackrel{\infty}{\infty}$ & ஸे & 㶽 & 0 & 织 & F & 品 \\
\hline$\frac{5}{\frac{5}{2}}$ & 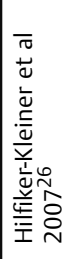 & & & 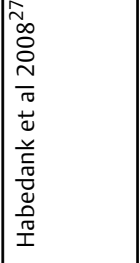 & 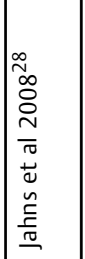 & 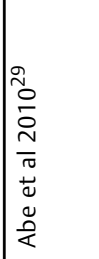 & $\begin{array}{l}\frac{0}{0} \\
\frac{1}{2} \\
\frac{\pi}{0} \\
\frac{0}{0}\end{array}$ & & & 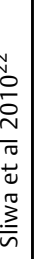 & & & & & & & & & \\
\hline & - & & & N & $m$ & \% & & & & 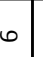 & & & & & & & & & \\
\hline
\end{tabular}




\begin{tabular}{|c|c|c|c|c|c|c|c|c|c|c|c|c|c|c|c|}
\hline 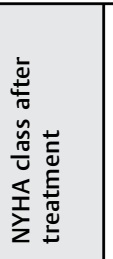 & 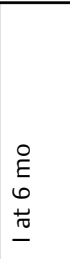 & 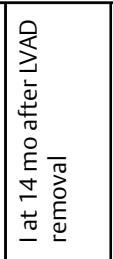 & 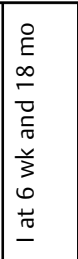 & $\frac{o}{z}$ & $\frac{\tilde{z}}{z}$ & $\frac{o}{z}$ & $\frac{\alpha}{z}$ & & $\frac{\alpha}{z}$ & $\begin{array}{l}\vec{\lambda} \\
\stackrel{v}{v} \\
-\end{array}$ & $\frac{o}{z}$ & $\begin{array}{l}2 \\
\overrightarrow{0} \\
0\end{array}$ & $\frac{\alpha}{z}$ & $\frac{\alpha}{z}$ & $\frac{\alpha}{z}$ \\
\hline 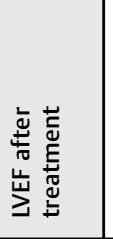 & $\begin{array}{l}\stackrel{\circ}{\leftrightarrow} \\
\stackrel{\circ}{+}\end{array}$ & 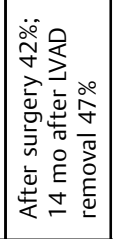 & 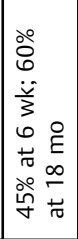 & 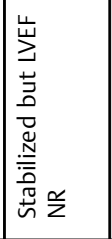 & 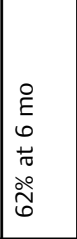 & $\frac{\alpha}{z}$ & 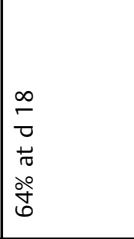 & & 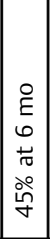 & 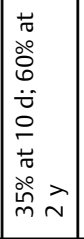 & 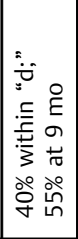 & 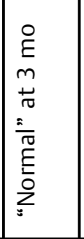 & 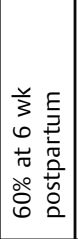 & 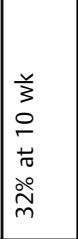 & 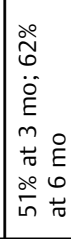 \\
\hline 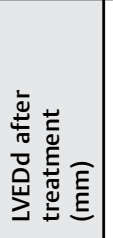 & i̊n & 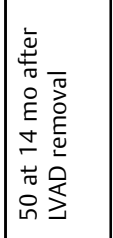 & $\frac{o}{z}$ & 兰 & $\frac{\alpha}{z}$ & 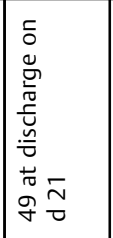 & 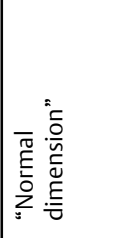 & & $\frac{x}{z}$ & $\frac{\alpha}{z}$ & $\frac{o}{z}$ & $\frac{\alpha}{z}$ & $\frac{\alpha}{z}$ & $\frac{o}{z}$ & $\frac{\mathscr{c}}{z}$ \\
\hline 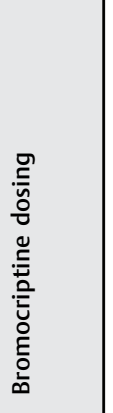 & 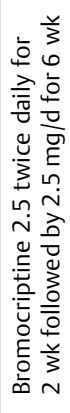 & 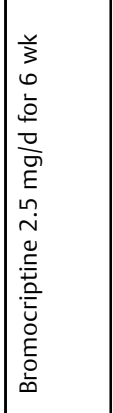 & 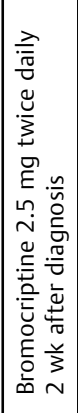 & 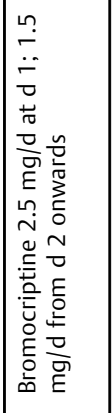 & 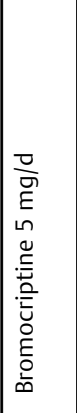 & 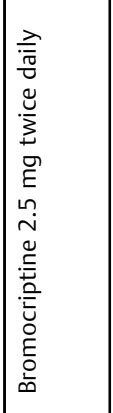 & 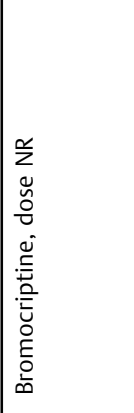 & & 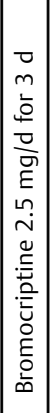 & 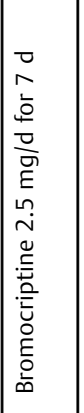 & 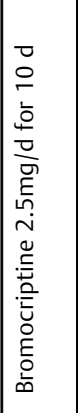 & 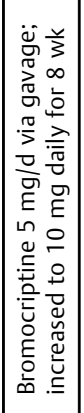 & 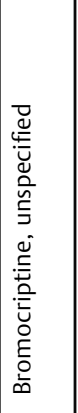 & 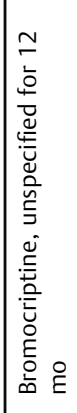 & 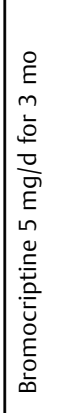 \\
\hline 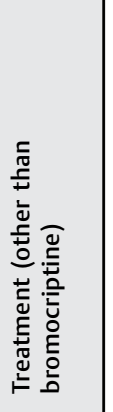 & 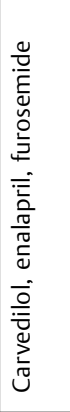 & 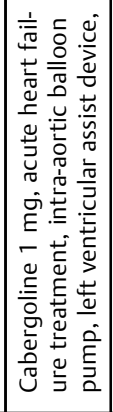 & 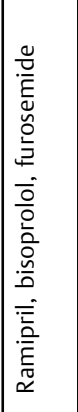 & 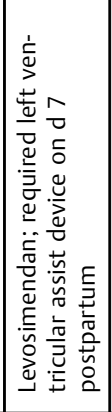 & 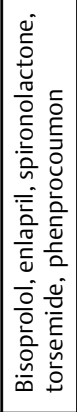 & 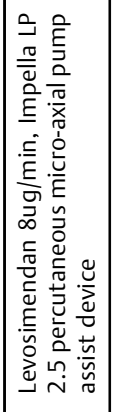 & 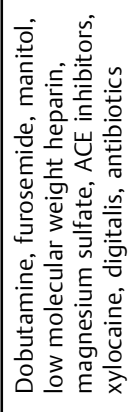 & & 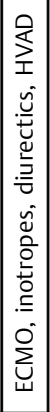 & 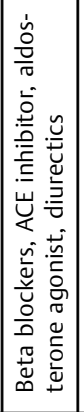 & 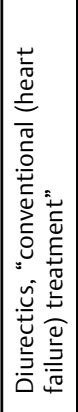 & 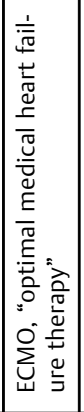 & 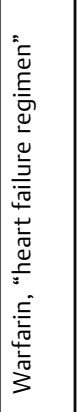 & $\frac{\alpha}{z}$ & 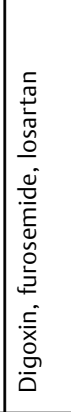 \\
\hline 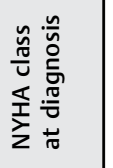 & $\equiv$ & 兰 & $=$ & $\geq$ & $\geq$ & $\frac{\alpha}{z}$ & $\frac{\tilde{c}}{z}$ & & $\frac{\tilde{z}}{z}$ & $\frac{\tilde{c}}{z}$ & $\equiv$ & $\frac{a}{z}$ & $\frac{o}{z}$ & 兰 & $\frac{\alpha}{z}$ \\
\hline 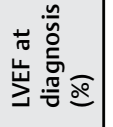 & $\infty$ & $\tilde{\sim}$ & $\stackrel{m}{m}$ & $\stackrel{\curvearrowleft}{\sim}$ & $\stackrel{\stackrel{N}{N}}{ }$ & 尔 & $\begin{array}{l}0 \\
\hat{1} \\
\stackrel{1}{n}\end{array}$ & & $\begin{array}{c}2 \\
\stackrel{1}{n} \\
\stackrel{n}{n}\end{array}$ & ळे & $\stackrel{\sim}{\sim}$ & $\frac{\alpha}{z}$ & 贞 & $\stackrel{1}{\stackrel{1}{\sim}}$ & q \\
\hline 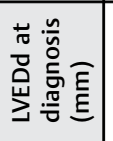 & 芦 & $\curvearrowright$ & $\frac{n}{2}$ & $\frac{\alpha}{z}$ & $\frac{\alpha}{z}$ & in & in & & $\frac{\mathscr{c}}{z}$ & $\frac{\mathscr{c}}{z}$ & $\frac{\mathscr{c}}{z}$ & $\frac{\mathscr{c}}{z}$ & & 孚 & $\frac{\alpha}{z}$ \\
\hline$\frac{5}{\frac{5}{2}}$ & & 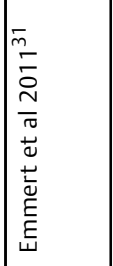 & 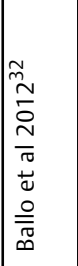 & 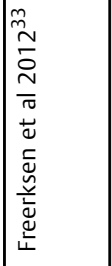 & 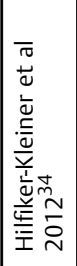 & $\mid$ & 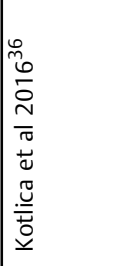 & 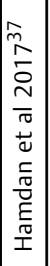 & & & & 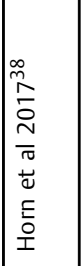 & 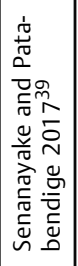 & 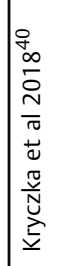 & 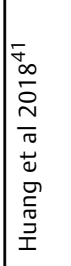 \\
\hline & & $\wedge$ & $\infty$ & $a$ & $\circ$ & $=$ & $\simeq$ & $1-1$ & & & & \pm & $\stackrel{2}{\sim}$ & 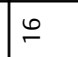 & $=$ \\
\hline
\end{tabular}


were case reports of patients with PPCMP treated with bromocriptine; these studies were included in this review.

Tabulation, Integration, and Results: Seventeen of these articles were case reports of patients with peripartum cardiomyopathy treated with bromocriptine that were included.

\section{Results}

We describe a review of the existing case studies from 2007 to 2018 that discusses use of bromocriptine in patients with PPCM ( - Table 2 and $\mathbf{3}$ ). These case reports describe the use of bromocriptine in 30 individual women, ranging in age from 18 to 43 years. The study subjects vary with respect to their country of origin, gravidities and parities, and gestational age. The onset of PPCM ranged from prior to delivery to as late as a month after delivery. The majority of these women recovered their left ventricular ejection fraction after receiving bromocriptine, typically dosed from 2.5 to $5 \mathrm{mg}$ daily, in conjunction with the standard heart failure therapy. Though many women presented with low ejection fractions, (range: 8-45\%), many were able to report NYHA classes II and I at time of follow-up.

While most of these are individual, heterogeneous case reports, 10 of these cases came from a pilot study comparing women with newly diagnosed PPCM receiving standard heart failure care $(n=10)$ versus standard care and bromocriptine $(n=10)$. This study demonstrated that the addition of bromocriptine to standard heart failure therapy improved NYHA functional class, left ventricular systolic and diastolic function, and degree of functional mitral regurgitation in women with PPCMP. Though this trial was small and far from definitive, the data appeared to show greater improvement in the group that received bromocriptine. Subsequently, a multicenter randomized controlled trial evaluated outcomes of 63 patients with PPCM who were treated with 1 or 8 weeks of bromocriptine in addition to standard therapy revealed that patients treated with bromocriptine was associated with higher rate of left ventricular recovery and had low morbidity and mortality. ${ }^{23}$ Post hoc analysis of this study demonstrated an improvement of the right ventricular function in addition to the left ventricular function at 6 month follow-up in women treated with bromocriptine. ${ }^{24}$ Bromocriptine may have a role in PPCMP patients with biventricular dysfunction. Addition of bromocriptine to the standard heart failure therapy, i.e. BOARD (Bromocriptine, Oral heart failure drugs, Anticoagulation, Relaxants [vasodilators for SBP $>110 \mathrm{~mm} \mathrm{Hg}$, Diuretics) has been proposed. Of note, prophylactic anticoagulation should be used when using bromocriptine to reduce the risk of thromboembolic complications. ${ }^{25}$

\section{Conclusion}

There is currently insufficient evidence for universal use of bromocriptine in addition to the standard treatment of PPCM. However, there are data to suggest that bromocriptine improves clinical outcomes. We recommend consideration of bromocriptine in selected cases of PPCMP. Future studies are indicated to elucidate its role as a standard therapy.
Précis

Bromocriptine seems to be a promising treatment for peripartum cardiomyopathy but there is a need for further clinical trials.

\section{References}

1 Arany Z, Elkayam U. Peripartum cardiomyopathy. Circulation 2016;133(14):1397-1409

2 Demakis JG, Rahimtoola SH. Peripartum cardiomyopathy. Circulation 1971;44(05):964-968

3 Demakis JG, Rahimtoola SH, Sutton GC, et al. Natural course of peripartum cardiomyopathy. Circulation 1971;44(06):1053-1061

4 Sliwa K, Hilfiker-Kleiner D, Petrie MC, et al; Heart Failure Association of the European Society of Cardiology Working Group on Peripartum Cardiomyopathy. Current state of knowledge on aetiology, diagnosis, management, and therapy of peripartum cardiomyopathy: a position statement from the Heart Failure Association of the European Society of Cardiology Working Group on peripartum cardiomyopathy. Eur J Heart Fail 2010;12(08):767-778

5 Kolte D, Khera S, Aronow WS, et al. Temporal trends in incidence and outcomes of peripartum cardiomyopathy in the United States: a nationwide population-based study. J Am Heart Assoc 2014;3(03):e001056

6 Brar SS, Khan SS, Sandhu GK, et al. Incidence, mortality, and racial differences in peripartum cardiomyopathy. Am J Cardiol 2007; 100(02):302-304

7 Bouabdallaoui N, Mouquet F, Lebreton G, Demondion P, Le Jemtel $\mathrm{TH}$, Ennezat PV. Current knowledge and recent development on management of peripartum cardiomyopathy. Eur Heart J Acute Cardiovasc Care 2017;6(04):359-366

8 Hilfiker-Kleiner D, Sliwa K. Pathophysiology and epidemiology of peripartum cardiomyopathy. Nat Rev Cardiol 2014;11(06):364-370

9 Desai D, Moodley J, Naidoo D. Peripartum cardiomyopathy: experiences at King Edward VIII Hospital, Durban, South Africa and a review of the literature. Trop Doct 1995;25(03):118-123

10 Yancy CW, Jessup M, Bozkurt B, et al; American College of Cardiology Foundation; American Heart Association Task Force on Practice Guidelines. 2013 ACCF/AHA guideline for the management of heart failure: a report of the American College of Cardiology Foundation/American Heart Association Task Force on practice guidelines. J Am Coll Cardiol 2013;62(16):e147-e239

11 Yancy CW, Jessup M, Bozkurt B, et al. 2016 ACC/AHA/HFSA focused update on new pharmacological therapy for heart failure: an update of the $2013 \mathrm{ACCF} / \mathrm{AHA}$ guideline for the management of heart failure: a report of the American College of Cardiology/ American Heart Association Task Force on clinical practice guidelines and the Heart Failure Society of America. J Am Coll Cardiol 2016;68(13):1476-1488

12 Bollen IA, Van Deel ED, Kuster DW, Van Der Velden J. Peripartum cardiomyopathy and dilated cardiomyopathy: different at heart. Front Physiol 2015;5:531

13 Amos AM, Jaber WA, Russell SD. Improved outcomes in peripartum cardiomyopathy with contemporary. Am Heart J 2006;152 (03):509-513

14 Fett JD, Sannon H, Thélisma E, Sprunger T, Suresh V. Recovery from severe heart failure following peripartum cardiomyopathy. Int J Gynaecol Obstet 2009;104(02):125-127

15 Elkayam U, Tummala PP, Rao K, et al. Maternal and fetal outcomes of subsequent pregnancies in women with peripartum cardiomyopathy. N Engl J Med 2001;344(21):1567-1571

16 Sliwa K, Fett J, Elkayam U. Peripartum cardiomyopathy. Lancet 2006;368(9536):687-693

17 Sliwa K, Förster O, Libhaber E, et al. Peripartum cardiomyopathy: inflammatory markers as predictors of outcome in 100 prospectively studied patients. Eur Heart J 2006;27(04):441-446 
18 Hilfiker-Kleiner D, Kaminski K, Podewski E, et al. A cathepsin Dcleaved $16 \mathrm{kDa}$ form of prolactin mediates postpartum cardiomyopathy. Cell 2007;128(03):589-600

19 Forster O, Hilfiker-Kleiner D, Ansari AA, et al. Reversal of IFNgamma, oxLDL and prolactin serum levels correlate with clinical improvement in patients with peripartum cardiomyopathy. Eur J Heart Fail 2008;10(09):861-868

20 Rayburn WF. Clinical commentary: the bromocriptine (Parlodel) controversy and recommendations for lactation suppression. Am J Perinatol 1996;13(02):69-71

21 Bernard N, Jantzem H, Becker M, et al; French Network of Regional Pharmacovigilance Centres. Severe adverse effects of bromocriptine in lactation inhibition: a pharmacovigilance survey. BJOG 2015;122(09):1244-1251

22 Sliwa K, Blauwet L, Tibazarwa K, et al. Evaluation of bromocriptine in the treatment of acute severe peripartum cardiomyopathy: a proofof-concept pilot study. Circulation 2010;121(13):1465-1473

23 Hilfiker-Kleiner D, Haghikia A, Berliner D, et al. Bromocriptine for the treatment of peripartum cardiomyopathy: a multicentre randomized study. Eur Heart J 2017;38(35):2671-2679

24 Haghikia A, Schwab J, Vogel-Claussen J, et al. Bromocriptine treatment in patients with peripartum cardiomyopathy and right ventricular dysfunction. Clin Res Cardiol 2018

25 Arrigo M, Blet A, Mebazaa A. Bromocriptine for the treatment of peripartum cardiomyopathy: welcome on BOARD. Eur Heart J 2017;38(35):2680-2682

26 Hilfiker-Kleiner D, Meyer GP, Schieffer E, et al. Recovery from postpartum cardiomyopathy in 2 patients by blocking prolactin release with bromocriptine. J Am Coll Cardiol 2007;50(24):2354-2355

27 Habedank D, Kühnle Y, Elgeti T, Dudenhausen JW, Haverkamp W, Dietz R. Recovery from peripartum cardiomyopathy after treatment with bromocriptine. Eur J Heart Fail 2008;10(11):1149-1151

28 Jahns BG, Stein W, Hilfiker-Kleiner D, Pieske B, Emons G. Peripartum cardiomyopathy-a new treatment option by inhibition of prolactin secretion. Am J Obstet Gynecol 2008;199(04):e5-e6

29 Abe T, Amano I, Sawa R, Akira S, Nakai A, Takeshita T. Recovery from peripartum cardiomyopathy in a Japanese woman after administration of bromocriptine as a new treatment option. J Nippon Med Sch 2010;77(04):226-230

30 Meyer GP, Labidi S, Podewski E, Sliwa K, Drexler H, HilfikerKleiner D. Bromocriptine treatment associated with recovery from peripartum cardiomyopathy in siblings: two case reports. J Med Case Reports 2010;4:80

31 Emmert MY, Prêtre R, Ruschitzka F, Krähenmann F, Falk V, Wilhelm MJ. Peripartum cardiomyopathy with cardiogenic shock: recovery after prolactin inhibition and mechanical support. Ann Thorac Surg 2011;91(01):274-276

32 Ballo P, Betti I, Mangialavori G, Chiodi L, Rapisardi G, Zuppiroli A. Peripartum cardiomyopathy presenting with predominant left ventricular diastolic dysfunction: efficacy of bromocriptine. Case Rep Med 2012;2012:476903

33 Freerksen N, Jaekel J, Menon AK, Maass N, Bauerschlag D. Massive respiratory dysfunction as sign of fulminant peripartum cardiomyopathy (PPCM). Hypertens Pregnancy 2012;31(04):451-453

34 Hilfiker-Kleiner D, Struman I, Hoch M, Podewski E, Sliwa K. 16-kDa prolactin and bromocriptine in postpartum cardiomyopathy. Curr Heart Fail Rep 2012;9(03):174-182

35 Schroeter MR, Unsöld B, Holke K, Schillinger W. Pro-thrombotic condition in a woman with peripartum cardiomyopathy treated with bromocriptine and an Impella LP 2.5 heart pump. Clin Res Cardiol 2013;102(02):155-157

36 Kotlica BK, Cetković A, Plesinac S, Macut D, Asanin M. Peripartum cardiomyopathy: a case of patient with triplet pregnancy. Clin Exp Obstet Gynecol 2016;43(02):274-275

37 Hamdan R, Nassar P, Zein A, Issa M, Mansour H, Saab M. Peripartum cardiomyopathy, place of drug therapy, assist devices, and outcome after left ventricular assistance. J Crit Care 2017;37:185-188

38 Horn P, Saeed D, Akhyari P, Hilfiker-Kleiner D, Kelm M, Westenfeld R. Complete recovery of fulminant peripartum cardiomyopathy on mechanical circulatory support combined with high-dose bromocriptine therapy. ESC Heart Fail 2017;4(04):641-644

39 Senanayake HM, Patabendige M. Two potentially lethal conditions of probable immune origin occurring in a pregnant woman: a case report. J Med Case Reports 2018;12(01):158

40 Kryczka KE, Dzielińska Z, Franaszczyk M, et al. Severe course of peripartum cardiomyopathy and subsequent recovery in a patient with a novel TTN gene-truncating mutation. Am J Case Rep 2018;19:820-824

41 Huang Y, Chen T, Zhang M, Yang X, Ding G, Yang L. Successful management of fatal peripartum cardiomyopathy in a young pregnant woman: a case report. Medicine (Baltimore) 2018;97 (15):e0408 